\title{
Publisher Correction: Combinatorial mutagenesis en masse optimizes the genome editing activities of SpCas9
}

Gigi C. G. Choi, Peng Zhou, Chaya T. L. Yuen, Becky K. C. Chan, Feng Xu, Siyu Bao D, Hoi Yee Chu DD, Dawn Thean, Kaeling Tan, Koon Ho Wong (D), Zongli Zheng (iD and Alan S. L. Wong (iD

Correction to: Nature Methods https://doi.org/10.1038/s41592-019-0473-0, published online 15 July 2019.

In the version of this Article originally published, Supplementary Fig. $2 \mathrm{c}$ was missing its data points. This has now been amended, and a replacement file uploaded. 